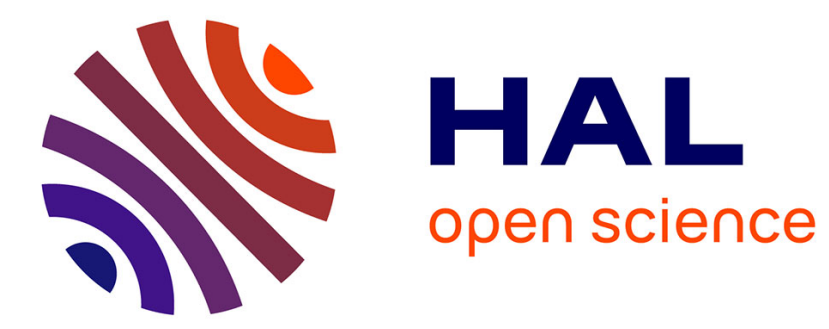

\title{
Une inscription inédite de Saillans (Drôme)
}

\author{
Henri Desaye
}

\section{To cite this version:}

Henri Desaye. Une inscription inédite de Saillans (Drôme). Gallia - Fouilles et monuments archéologiques en France métropolitaine, 1984, 42 (2), pp.233-241. 10.3406/galia.1984.1920 . hal01941038

\section{HAL Id: hal-01941038 \\ https://hal.science/hal-01941038}

Submitted on 27 Feb 2020

HAL is a multi-disciplinary open access archive for the deposit and dissemination of scientific research documents, whether they are published or not. The documents may come from teaching and research institutions in France or abroad, or from public or private research centers.
L'archive ouverte pluridisciplinaire HAL, est destinée au dépôt et à la diffusion de documents scientifiques de niveau recherche, publiés ou non, émanant des établissements d'enseignement et de recherche français ou étrangers, des laboratoires publics ou privés.

\section{(이) $\$$}

Distributed under a Creative Commons Attribution - NonCommercial - NoDerivatives| 4.0 


\title{
NOTE
}

\section{UNE INSCRIPTION INÉDITE DE SAILLANS (Drôme)}

\author{
par Henri DESAYE
}

En creusant une tranchée le long du chemin vicinal dit Route royale, sur la parcelle B 488, au quartier de la Maladrerie, à Saillans (26430), le 12 mai 1982, la pelle mécanique faisail découvrir une grande table de marbre représentant la partie gauche d'une inscription. Elle reposait verticalement au pied d'une petite butte et sa face écrite était recouverte d'une légère pellicule de tartre ${ }^{1}$.

L'inscription était donc portée par deux plaques de marbre de dimensions semblables : long. : 1,36 m ; haut. : $1,04 \mathrm{~m}$; épais. : 0,275 m. Elles étaient à l'origine juxtaposées, l'une à gauche, l'autre à droite. Plusieurs trous se reconnaissent sur la tranche supérieure de chaque plaque (fig. 1). Au centre, un trou allongé ; symétriques par rapport à lui, deux entailles prolongées en direction de l'arrière par une rainure; enfin, trois petits trous carrés, deux près des extrémités, symétriques, et un près du milieu, mais vers l'arrière.

Cette variété semble correspondre à des usages différcnts. L'ouvertur'e centrale allongée paraît avoir été destinée à la manutention de la pierre. Les deux entailles à rainures devaient recevoir le scellement des crampons qui liaient la plaque à d'autres gros blocs situés derrière.

1 l'rivenus immedialement par les entrepreneurs, MM. Georges Granon et Jean Gautheron, qui aflirmaient. qu'une seconde plaque avait éte aperçue dans la tranchée, nous pùmes organiser, avec le concours de la Direction régionale des antiquités historiques, un sauvetage. L'ensemble, depuis, a été donné par M. Granon à la commune de Saillans.
Le petit trou latéral le plus proche de la plaque voisine a reçu un scellement en plomb qui fixait un crampon de fer, assujettissant entre eux les deux éléments de l'inscription, ce que confirment des traces bien visibles de rouille et même la présence de plomb. L'autre petit trou symétrique pouvait lier la plaque à un bloc placé immédiatement à gauche ou à droite en façade. Nous ignorons l'utilité du dernier petit trou.

Sur la tranche inférieure (base), on remarque un entaille profonde à l'angle droit de chaque plaque, l'angle inférieur gauche de la plaque de droite étant brisé. Le scellement des marbres paraît avoir été assuré de façon moins solide en bas qu'en haut.

Les tranches des plaques sont ciselées, montrant à la fois des zones lisses plus ou moins importantes près des bords et des parlies où l'on reconnait la trace des coups de ciseaux. Le derrière n'est que dégrossi.

M. François Braemer, vice-président, du Corpus des sculptures de l'Empire romain, a bien voulu nous indiquer que le matériau était un marbre du type de l'Apennin provenant d'une carrière de la région de Carrare et préciser que l'on exploitait ce faciès au ier siècle.

Une moulure en doucine, faisant une saillie de $0,025 \mathrm{~m}$ sur le champ épigraphique, entoure le texte. La surface de ce champ épigraphique ne se montre pas plane, mais légèrement convexe, mouvement qu'adoptent également les moulures horizontales. Pour chaque plaque, la convexité forme une saillie de 0,018 à $0,022 \mathrm{~m}$ (flèche de la corde de l'arc). On peut 


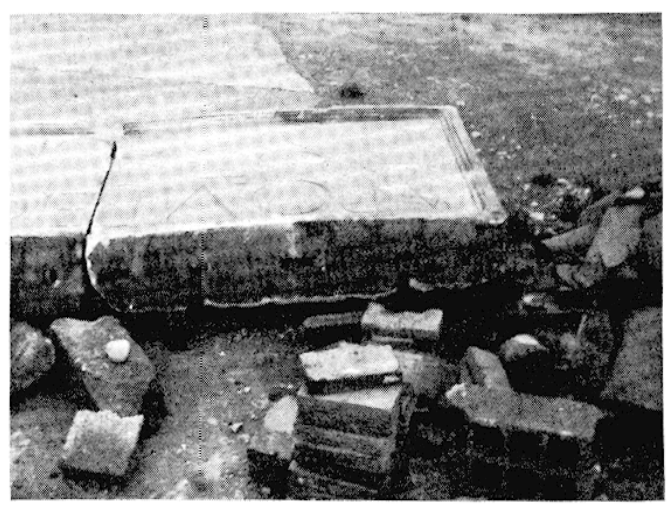

1 L'inseription de Saillans. Table de marbre double. Trous de scellement sur la tranche supérieure de la première plaque de marbre.

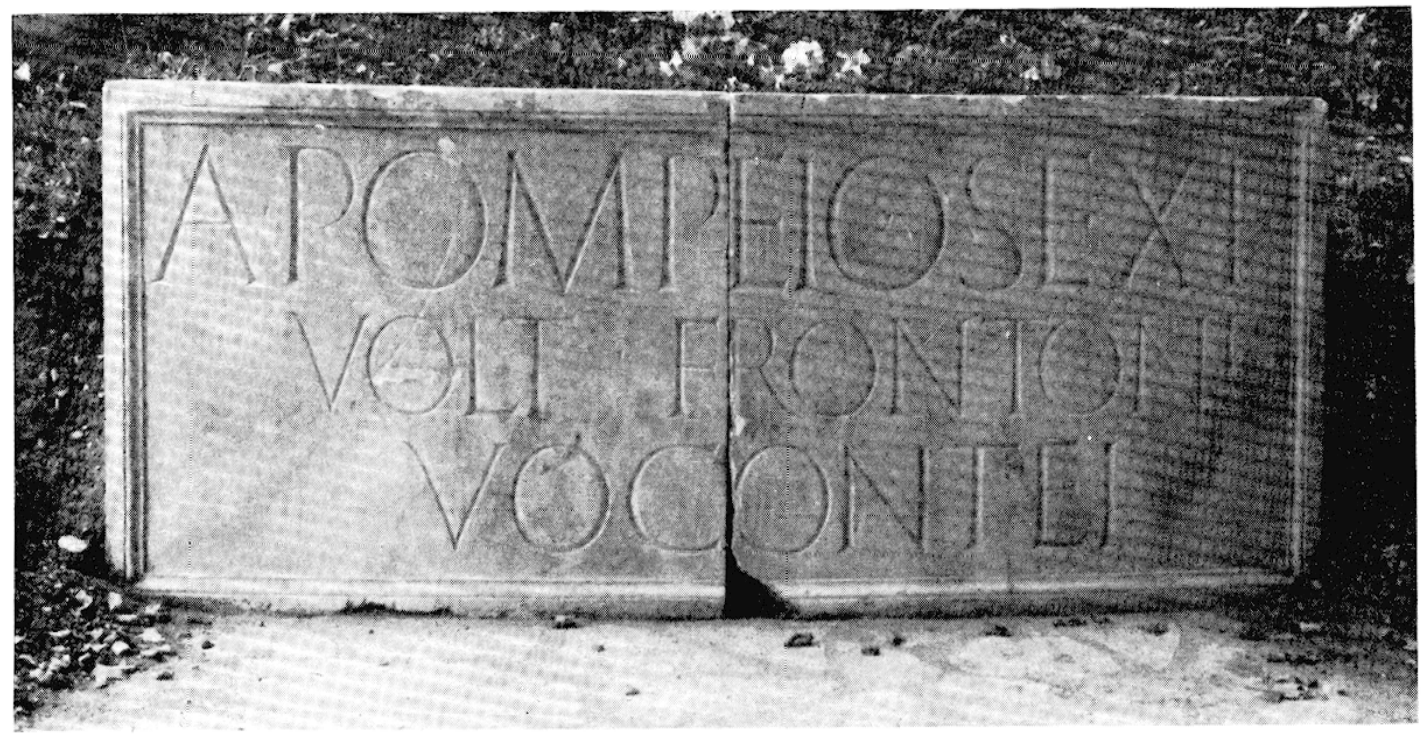

2 L'inscription de Saillans.

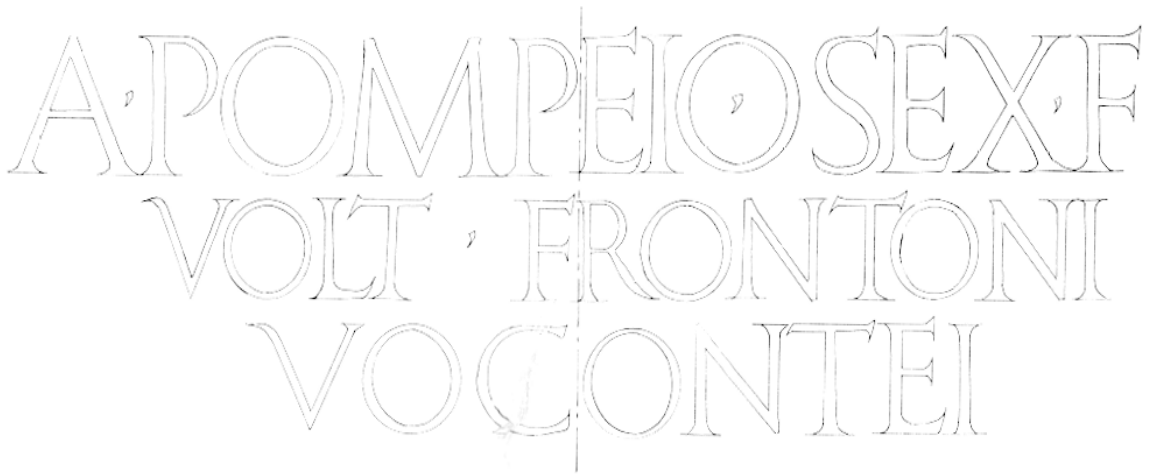

3 Relevé de l'inscription d'.uprès frottis. Relevé et dessin P'. Bellier. 
tenter de calculer à partir de cette courbure le diamètre d'un monument circulaire, malgré les risques d'erreur : on obtient, un rayon se siluant entre 11 et $13 \mathrm{~m}$, ce qui représente un monument important.

I'inscription est gravée en belles lettres de respectivement $0,277 \mathrm{~m}, 0,20 \mathrm{~m}$ et $0,223 \mathrm{~m}$ de haut (fig. 2 el 3 ). On observe des traces de réglures au haut et au bas des lignes. Ie ductus ne trahit aucune raideur : on le remarque notamment dans la queue du $R$, dont le plein s'amincit et s'incurve délicatement et brièvement en touchant la ligne de base. De même la branche gauche du $\mathrm{X}$ et des deux V s'amorce en haut à partir d'un empattement selon un tracé incurvé. Les pleins et les déliés sont toujours marqués, y compris dans les 0 , larges et circulaires. Le $\mathrm{M}$ n'a pas les jambages parallèles deux à deux. Ni le $A$, ni le $M$, ni les trois $\mathrm{N}$ ne présentent des extrémités supérieures pointues, mais la rencontre des jambages ascendants et descendants se marque par une oblique due à la prédominance du plein descendant sur le délié ascendant, selon le ductus naturel. Pas de compénétrations de lettres non plus, ni de $\mathrm{T}$ dépassant la ligne en haut.

Ainsi donc on n'observe pas ici quelques-unes des caractéristiques de l'écriture monumentale augustéenne, et notamment la raideur, comme on en a des exemples en Gaule au temple de Valetudo élevé à Glanum par Agrippa ${ }^{2}$, au mausolée des Jules ou du sévir lyonnais Q. Galvius Turpio ${ }^{3}$. En revanche, le marbre de Saillans présente dans son écriture quelques marques d'ancienneté : les $O$ sont circulaires, les deux $F$ ont des branches horizontales presque égales, le $\mathrm{E}$ de la troisième ligne possède une barre horizontale inférieure nettement plus longue que les deux autres avec un empattement marqué et bien relevé; cet allongement correspond à une tendance, reconnue dans plusieurs textes d'époque julio-claudienne che\% les Voconces seplentrionaux, qui consiste à placer un empattement important et très relevé

2 Henri Rolland, Fouilles de Glanum, 1947-1956, XIe suppl. à Gallia, 1958, p. 103-104 et pl. 36, 3.

3 CIL XII 1012 ; XIII 1941. au pied des hastes ou à l'extrémité des branches du $E^{4}$.

Les points n'ont pas, à Saillans, la forme d'un simple triangle, mais d'un triangle dont la partie inférieure se prolonge par une sorte de virgule tournée à gauche. Les textes des Voconces du Nord semblent bien montrer que, même dans le domaine de la ponctuation, il se produit dès le II $^{\mathrm{e}}$ siècle un tarissement de l'imagination et une raréfaction des formes originales ou élégantes. Enfin et surtout le point inséré dans le 0 , pour marquer ici la fin du mot Pompeio, se rencontre, selon Cagnat ${ }^{5}$, à l'époque de Gésar; on continue à le trouver sous Auguste et les premiers JulioGlaudiens, comme le confirment des textes d'Arles, de Valence, de Vienne, de Lyon, de Bordeaux ${ }^{6}$, à défaut d'un recensement complet en Narbonnaise. On notera, en passant, que sur le marbre de Saillans il n'y a pas de point à la fin des lignes, puisqu'aucun mot n'empiète et qu'il ne peut exister de difficultés de lecture.

La mise en page est soignée, bien que le lapicide ne soit pas arrivé à laisser pour chaque ligne une marge égale à gauche et à droite : on le voit bien à la fin de la seconde ligne, un peu serrée. Cela vient de ce qu'il n'était guère possible de graver une lettre dans l'axe même du texte, car elle se serait trouvée à cheval sur les deux plaques.

$$
\text { ** }
$$

La lecture du texte ne fait aucune difficulté :

$$
\begin{gathered}
\text { A(ulo). Pompeio. Sex(ti) .f(ilio) ! } \\
\text { Volt(inia). Frontoni / Vocontei }
\end{gathered}
$$

"A Aulus Pompeius Fronto, fils de Sextus, de la tribu Voltinia, les Voconces (ont fait ce monument) is.

4 Cf. notamment $G I L$ XII 1579 et $I L G N 243$.

5 Cours d'épigraphie latine, $3^{\text {e }}$ éd., Paris 1898, p. 19, n. 3 .

6 Arles : CIL XII 762; Valence : André Blanc et Henri Desaye, Inscriptions nouvelles de la Drôme el de l'Ardèche, dans Gallia, 33, 1975, p. 231-232; Vienne : $C I L$ XII 1902 (après 36-41) et Yves BurNand, Chronologie des epitaphes romaines de Vienne, dans Revue des Etudes Anciennes (abrégé : REA), LXIII, 1961, p. 295; Lyon : CIL XIII 1769 (sous Tibère) et Amable Audin et Yves Burnand, Chronologie des épilaphes romaines de Lyon, dans REA, LXI, 1959, p. 328 ; Bordeaux : CIL XIII 569. 
L'onomastique du personnage désigné ici est entièrement romaine, ne comportant aucun élément indigène. Cependant l'inscription dans la tribu Voltinia, qui est celle des cités latines de Narbonnaise, laisserait croire que A. Pompeius Fronto, citoyen romain, appartenait à l'une d'entre elles. On pense même tout naturellement à la cité des Voconces sur le territoire de laquelle se trouvait le monument de Saillans offert par ses citoyens ${ }^{7}$. En principe, la filiation par le prénom du père tendrait à indiquer que ce dernier avait déjà la citoyenneté ou, du moins, portait déjà un nom conforme à l'onomastique des citoyens romains.

Il y a peu à dire sur le cognomen Fronto, bien attesté en Narbonnaise (50 exemples d'après les tables de $C I L \mathrm{XII}$ ) et même signalé à Vaison ${ }^{8}$. Les prénoms paraissent intéressants : Aulus et surtout Sextus apparaissent fréquemment et depuis longtemps dans la gens Pompeia, le premier en particulier dans la lignée des Bithynici, le second dans celle des Magni. Le plus célèbre des personnages qui aient porté ce dernier prénom est évidemment le fils du grand Pompée; un Sex. Pompeius, fils de Sextus consul en 5 av. J.-G., petit-fils d'un autre Sextus consul en 31 av. J.-G., a été gouverneur de Macédoine en 8-9, puis consul en 14. Et il en est d'autres! Le prénom Sextus apparaît 13 fois en Narbonnaise devant le gentilice Pompeius et Aulus 10 fois selon les indices du CIL.

On connaît même dans la province plusieurs Pompeius Fronto. Deux (ou trois) portent le prénom, très pompéien, de Gnaeus : l'un est mentionné sur l'arc de Gavaillon, l'autre est apparitor et s'acquitte à Nîmes d'un vœu à Junon; le troisième, s'il s'agit bien d'un

7 Plusieurs textes se rapportant à des Voconces du Nord mentionnent la lribu voltinia : CIL XII 1579, 1661 ; FOR XI, app. épigr. 65 ; Gallia, XVIII, 1960, p. 205-206; CIL III 1653, 11223 ; XIII 6882, 6978, 7011, 7013, 8059, 8060. Chr. Goudiveav, Les fouilles de la maison au dauphin à Vaison, XXXVIIc suppl. à Gallia, 1979, p. 264-270, altribue à César la concession du droit latin à Vaison et à Luc.

8 CIL XII 1303 : Q. Abudius Frontonis l(ibertus) Theodotus.

9 W.-H. Gross, Pompeius, dans Paulys RealEncyclopädie der classischen Altertumswissenschaft (abrégé : $R E$ ), 21 II, col. 205I-2052 et nos 1-4, 16-20, 33, $61,62,115$. troisième, est connu par un texte trouvé $\dot{a}$ Marguerittes à quelques kilomètres de Nîmes ${ }^{10}$. Enfin, sur la colline Saint-Jacques au-dessus de Cavaillon, une inscription, que Calvet attribuait au début de l'époque d'Auguste, cite quatre quattuorvirs, de Cavaillon évidemment, dont A. Pompeius Fronto, fils de Sextus, quattuorvir pour la seconde fois ${ }^{11}$. C'est exactement le nom de Saillans. Est-ce à dire qu'ii s'agisse du même personnage? Malgré la coïncidence troublante du nom, de la filiation et sans doute de l'époque, ce n'est pas évident, vu la fréquence du gentilice Pompeius accompagné des prénoms Aulus, Sextus ou Cnaeus et celle du cognomen Fronto. Les deux Cn. Pompeius Fronto de Cavaillon et de Nîmes montrent bien la banalisation extrême, dans une province aussi romanisće que la Narbonnaise, des noms et prénoms rappelant les grands personnages de la République. Il n'y a pas lieu de s'en étonner quand on sait que transmettaient leur nom et prénom ceux qui faisaient obtenir la citoyenneté comme ceux qui affranchissaient.

A propos précisément du gentilice Pompeius, très répandu en Narbonnaise (147 Pompeius et 88 Pompeia en $C I L$ XII et $I L G N)$, on peut se demander comment notre personnage de Saillans a reçu ce nom et, en fin de compte, qui il était. Certes il peut s'agir d'un Italien établi dans la région comme négociant ou propriétaire foncier, mais, on l'a vu, on pense plutôt à un indigène dont le père ou un ancêtre aurait reçu la cité romaine par l'entremise d'un membre de la gens Pompeia. Il parait vain de rechercher quel Pompeius a pu faire obtenir cette faveur ; remonter au grand Pompée lui-même reste une pure hypothèse. On ne peut conclure d'un passage de Justin ${ }^{12}$ que Pompće, aprc̀s la dćfaitc de Scrtorius, ait continué en Gaule la politique de clémence qu'il avait inaugurée en Espagne ${ }^{13}$. Le texte

10 CIL XII 1056, 3062, 3011.

11 CIL XII 1051: A(ulus) Pompeius Sex(ti) f(ilius) / Fronto iler(um) Sex(tus) / Lucceius A(uli) f(ilius) [Ae]lianus / $D$ (ecimus) Iulius $D$ (ecimi) f(ilius) Labeo / C(aius) Areius C(ai) f(ilius) Fronto / IIII uir(i) f(aciendum) c(uraverunt) ।

$1243,5,11$.

13 Comme l'ont fait CIL XII, p. 160; Camille Jullisn, Hisloire de la Gaule, III, p. 116 ; Joseph 
ne parle que d'un cas particulier, celui du grandpère voconce de l'historien Trogue Pompée, qui reçut la cité du général romain, et il serait abusif d'étendre à toute une partie de l'aristocratie locale cette concession. Comme le pense Chr. Goudineau, c'était là une faveur personnelle que faisait Pompée à un homme qui avait servi sous ses ordres lors de la guerre contre Sertorius ${ }^{14}$.

Le gentilice Pompeius-a est porté par 20 personnes de la cité des Voconces, mentionnées dans 18 textes. Par rapport aux 235 membres de la gens connus en $C I L$ XII et $I L G N$, cela représente $8,51 \%$. Si l'on pense que les
Voconces ont livré, d'après ces deux recueils, 533 inscriptions sur un total de 6214 pour la Narbonnaise, soit $8,57 \%$, on admettra que le nombre des Voconces parmi les Pompeii correspond exactement à l'importance de l'épigraphie de cette cité dans l'ensemble de la province : nous sommes ici dans la moyenne. Si, d'autre part, on recherche le pourcentage de Pompeii-ae par rapport au nombre d'inscriptions trouvées chez les Voconces, on arrive à $3,75 \%$, proportion tout à fait honorable $e^{15}$.

Voici la liste des 18 textes voconces (Vaison, Luc, Die, Bochaine) se rapportant à la gens Pompeia :

\begin{tabular}{|c|c|c|c|c|}
\hline RÁÝRERNCE: & Provenaxce & NATURE & DATE & Nom \\
\hline CIL 1311 & Vaison & tinurobole, perdu & fin $\mathrm{Ir}^{\mathrm{e}}-\mathrm{III}^{\mathrm{e}} \mathrm{s}$ & A. Pompeius Avitianus \\
\hline 1371 & Entrechaux & cippe funéraire, datif & $70-100$ & $\begin{array}{l}\text { Q. Pompeius ... Volt., édile, pré- } \\
\text { fet, prétcur, flamine, pontife; } \\
\text { Pompeia S... }\end{array}$ \\
\hline 1372 & Vaison & datif, perdu & I $^{\mathrm{er}} \mathrm{S}$. ? & $\begin{array}{l}\text { Q. Pompeius Volt. Titi ? f. Red? } \\
\text { flamine }\end{array}$ \\
\hline 1438 & Vaison & $\begin{array}{l}\text { funéraire, filiation par le pré- } \\
\text { nom, perdu }\end{array}$ & $\mathrm{I}^{\mathrm{er}}-\mathrm{II}^{\mathrm{e}} \mathrm{S} . ?$ & Pompeia... \\
\hline 1453 & Vaison & perdu & $?$ & Pompeia Festa \\
\hline 1464 & Vaison & $d m$, perdu & ? & Pompeia Demetria \\
\hline 1531 & $\begin{array}{l}\text { Bâtie-Yont- } \\
\text { Saléon }\end{array}$ & $\begin{array}{l}\text { dédicace à Allobrox, dédicant }+ \\
\text { dieu }\end{array}$ & ier $\mathrm{s}$. & Pompeia Lucilla \\
\hline 1560 & Die & dédicace à Andarta, bonnes lettres & $\mathrm{II}^{\mathrm{e}} \mathrm{s}$. & M. Pomp(cius) Primitivus \\
\hline 1573 & Luc & perdu & Ier s.? & ... r Pomp(eius) Luc..., prêtre? \\
\hline 1594 & Dic & bandeau, $d m$, bonnes lettres & Ire moitié In $\mathrm{s}$ & Pomp(eia) Iphigenia \\
\hline 1638 & Die & stèle, $d m$, tendance à la rustique & $\mathrm{II}^{\mathrm{e}} \mathrm{s}$. & Sex. Pomp(eius) Eutyches \\
\hline 1649 & Die & table, $d m$, lettres médiocres & III ${ }^{e} \mathrm{~s}$. & $\begin{array}{l}\text { L. Pomp(eius) Faustinus, L. } \\
\text { Pomp(eius) Hermeros }\end{array}$ \\
\hline 1650 & Luc & grand bandeau funéraire, perdu & $\mathrm{I}^{\mathrm{er}}$-début $\mathrm{II}^{\mathrm{e}} \mathrm{s}$. & Pomp(eius) $\vee \ldots$ \\
\hline 1651 & Die & perdu & ? & Pompeia Eutychia \\
\hline 1664 & Die & stèle, $d m$, lettres correctes & 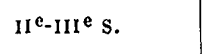 & Pompeia Servatilla \\
\hline 1670 & Die & perdu & $?$ & C. Pompeius Valerianus \\
\hline \multirow[t]{2}{*}{$I L G N 243$} & Die & slèle, datif, écriture ancienne & Ier $\mathrm{s}$. & Q. Pompeius Filo \\
\hline & Saillans & $\begin{array}{l}\text { plaques marbre, datif, lettres } \\
\text { excellentes }\end{array}$ & $\begin{array}{l}2 \text { e moitié prin- } \\
\text { cipat d'Auguste }\end{array}$ & A. Pompeius Sex. f. Volt. Fronto \\
\hline
\end{tabular}

Sauter, Vaison dans l'anliquilé, Avignon 1926, I, p. 112-113.
14 Chr. Goudineau, op. cil., p. 252-253.

15 op. cil., p. 254. 
Il faudrait ajouter à ce tableau trois textes où Pompeius-a n'est plus employé comme gentilice, mais comme cognomen :

\begin{tabular}{|c|c|c|c|c|}
\hline RÉFÉRENCE & Provenance & Nature & Date & Nou \\
\hline CIL 1414 & Vaison & datif funéraire, perdu & Ier $\mathrm{s}$. & Iulius Pompeius \\
\hline 1629 & Dic & stèle-cippe, $d m$, belles lettres & 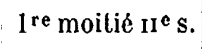 & Iulia Pompeia \\
\hline 1650 & Luc & grand bandeau funéraire, perdu & $\mathrm{I}^{\mathrm{er}}$-début II $\mathrm{e}$. & ... T. fil. Pompeia \\
\hline
\end{tabular}

Enfin on ne sait si Pompeia était gentilice ou cognomen sur le fragment qui a donné son nom au Portique de Pompée à Vaison ${ }^{16}$.

Ces cognomina pouvaient rappeler un genlilice utilisé par un ascendant ou avoir été un ancien nom unique ; les trois exemples voconces paraissent remonter assez haut.

Tous les gentilices Pompeius-a ne peuvent être mis sur le même plan. Pompeia Demetria, Sex. Pompeius Eutyches, Pompeia Eutychia, Q. Pompeius Filo, L. Pompeius Hermeros, Pompeia Iphigenia paraissent être des affranchis ou des descendants d'affranchis ; tous les hommes ont les tria nomina. Pompeius Luc... et Pompeius V..., l'un et l'autre de Luc-enDiois, ont, eux, déjà abandonné le prénom, si du moins les textes ont été bien lus, et cela probablement à époque assez ancienne. Seuls trois personnages indiquent leur filiation : on remarquera que tous les trois mentionnent leur appartenance à la tribu Voltinia.

Les prénoms employés sont Quintus (3), Sextus (2), Aulus (2), Lucius (2), Caius (1), Marcus (1), Titus? (1), les prénoms traditionnels dans la gens - les trois premiers - étant les plus nombreux et les seuls usités au $\mathrm{I}^{\mathrm{er}}$ siècle.

On s'apercoit que, sur les 18 textes cités, 7 - c'est-à-dire plus du tiers -- peuvent remonter à ce $\mathrm{I}^{\mathrm{er}}$ siècle. Si les gentilices durent longtemps grâce à l'hérédité, à l'adoption, à l'affranchissement et à l'octroi de la citoyenneté, ils ont quand même tendance à s'user, ainsi que les prénoms usuels dans une gens, et les grands noms de la République s'effacent à la fin du ${ }^{\mathrm{e}}{ }^{\mathrm{e}}$ siècle au profit d'autres plus récents.

16 J. Sautel, op. cit., II, suppl. p. 11.
On est d'autre part frappé de constater que le gentilice Pompeius est bien plus attesté dans la vallée de la Drôme (11 textes) que dans la région même de Vaison ( 6 textes). Et, sans avoir le ridicule de supposer que Trogue Pompée était originaire du Diois, peut-être faudrait-il ne pas estimer que seule la portion méridionale de la cité brillait de quelque éclat au début de l'Empire : le marbre de Saillans peut contribuer à une vision plus juste et plus équilibrée des choses.

I a fin de l'inscription contient la forme archaïque Vocontei, inconnue jusqu'ici, mais asse\% facile à expliquer. Il s'agit du nominatif pluriel de l'ethnique Vocontius. La diphtongue $e i$, conforme dans ce cas à l'étymologie, s'est réduite à la voyelle $i$ conformément aux lois de la phonétique latine, mais la graphie ei s'est maintenue en concurrence avec $i$ jusqu'au début de notre ère, où $i$ l'a emporté définitivement $^{17}$. Vocontei est donc l'équivalent, avec une orthographe archaissante, de la forme Voconti. Or Voconti résulte de la contraction de $i$ avec la voyelle $i$ : ainsi trouve-t-on à Valence le nominatif pluriel Cassi ${ }^{18}$ et l'épigraphie fournit de nombreux exemples semblables ${ }^{19}$. Dans l'écriture, la forme ii l'a emporté grâce aux grammairiens qui soutenaient que le nominatif pluriel devait avoir le même nombre de syllabes que le nominatif singulier ${ }^{20}$. A Saillans la contraction s'est faite normalement, mais alors que se maintenait

17 A. Ersout, Morphologie historique du latin: Paris, nouv. éd. 1941 , p. 50-51.

18 CIL XII 1746.

19 Jules Pirson, La langue des inscriptions latines de la Gaule, Bruxelles 1901, p. 117.

20 A. ErnouT, op. cil., p. 51. 
l'ancienne graphie ei. De même au Pont Flavien, à Saint-Chamas, rencontre-t-on les génitifs singulier Donnei et Altei, dans lesquels le $i$ est contracté avec le $e i$, la diphtonguc n'étant ici qu'une manière de transcrire le $\bar{i}^{21}$.

Les lextes dans lesquels on rencontre l'orthographe ei sont, d'après les tables de CIL XII et XIII, 30 en Gaule. Tous, sauf $~^{22}$, ont été trouvés en Narbonnaise; encore sont-ils rares en Narbonnaise du Nord ${ }^{23}$. La plupart proviennent de cités très anciennement romanisées, Narbonne en particulier et les cités de la basse vallée du Rhône ${ }^{24}$. On peut presque tous les attribuer avec sûreté au début de l'époque julio-claudienne. Par exemple, on y remarque les formules heic quiescit, heic sepultus est, heic silus est écrites en entier ${ }^{25}$, la raideur des lettres (nous avions déjà signalé à ce propos les mausolées des Jules et du sévir Turpio), l'absence presque totale de caractéristiques récentes ${ }^{26}$; une date consulaire fait remonter CIL XII 2693 à 8 av. J.-C. Des textes mentionnent un sévir et un flamen Romae et Augusli $^{27}$ : par les sacerdoces qu'ils rappellent, ils ne peuvent être antérieurs à l'organisation du culte impérial en Gaule.

J)ans ces conditions, nous ne pensons pas être trop loin de la vérité en disant que le marbre de Saillans, compte tenu de la souplesse et de la perfection de son écriture, remonte a la seconde moitié du principat d'Auguste.

Cette inscription néanmoins pose deux questions.

Devant la beauté de la gravure, on croira difficilement, comme nous l'a fait observer notre ami Jean Pietri, que ce soit là l'œuvre

\section{CIL XII 647.}

22 GIL XIII 1394, 1941, 2333, 7028, 8249.

23 CIL XII 2665 (le Pouzin), 1918 (Vienne), 2598, 2600,2623 (Genève).

24 Narbonne : CIL XII 4455, 4491, 4780, 4883, 4906, 4933, 4947, 4978, 5095, 5102, 5252; Arles et son territoire : $647,744,870$; Glanum : 1012 ; Nîmes : 3369 ; Avignon : 1038; Carpentras : 1164; Orange: 1262 ; Vaison : 1340.

25 CIL XII 870, 1262, XIII 2333; XII 4455, 4491, 4933, 4978; 1038.

26 On trouve cependant la graphie vivus : CIL XII 3369 ; l'age du défunt : 1918 (mais I $\lambda$ xpse est une transcription grecque), XIII 7028 ; la formule $a \mathrm{~m} \mathrm{el}$ memoriae : XIII 1394.

27 CIL XIII 1941 ; XII 647 et 2600. d'un artiste local, de Luc ou de Valence. Faut-il imaginer que le texte a été gravé en Italie, près de la carrière même, par des lapicides hautement entrainés? Cela expliquerait l'absence de tout archaïsme dans la forme des caractères.

Plus étonnante encore la brièveté du texte. Que l'on ait affaire à une épitaphe ou à un texte honorifique, on ne peut qu'être surpris de n'y voir figurer ni les titres et qualités du personnage -.-il n'y a strictement que son nom - ni les raisons qui ont poussé les Voconces à élever le monument. L'explication la plus simple consisterait à supposer qu'il existait à proximité une autre inscription, plus développée, contenant tout ce que le texte que nous possédons a omis, peut-être dans un souci de monumentalité et de sobriété augustéennes.

La dédicace est faite par les Voconces tout court : il n'est question ni de la res publica, ni de l'ordo, ni de la civitas ${ }^{28}$. C'est là une manière de faire plus ancienne, qui ignore le système politique romain. On n'en connait pas d'autres exemples chez les Voconces, les dédicaces des Vasienses Vocontii s'expliquant peut-être par la difficulté de trouver un terme juridique exact pour désigner cette fraction du peuple 29 .

Il paraît inutile de rappeler qu'à Saillans nous sommes bien sur le territoire des Voconces, qui s'étendait sur toute la vallée de la Drôme en amont de Crest et d'Aoustesur-Sye : le confirment les limites médiévales du diocèse de Die et, surtout, les milliaires de Saillans qui donnent une distance calculée à partir de Dea Augusta ${ }^{30}$.

\section{$\therefore$}

Les autres trouvailles faites sur le site de la Maladrerie nous fournissent quelques renseignements intéressants, mais d'interprétation parfois difficile.

28 Res publica Voconliorum: ILGN 231 (annéc 208; ; ordo Vocontiorum ex consensu et postulatione populi : CIL XII $1585\left(2^{\mathrm{e}}\right.$ moitié du I $^{\text {er-début }}$ du ${ }_{1 I^{\mathrm{e}}} \mathrm{s}$.); civitas Vocontiorum : 1567 (année 245).

29 CIL XII 1357, 1360 = 5842; ILGN 207. Le mot res publica était en lui-même ambigu : cf. XII 1357 : res publica Iuliensium.

30 CIL XII 5504 et 5504 a. 
Il existait à cet endroit un grand monument, dont les vestiges étaient assez impressionnants pour que l'endroit s'appelât au xvre siècle Ad Romanon et au xvir ${ }^{\mathrm{e}} \mathrm{Pas}$ de Romanon ${ }^{31}$ et que Chorier plaçât à Saillans un arc de triomphe commémorant la victoire de Marius sur les Teutons ${ }^{32}$. Vers le milieu du xix ${ }^{e}$ siècle, on y apercevrait encore une masse de maçonnerie sur le bord d'un petit ruisseau qui traverse la route ${ }^{33}$. Plusieurs fragments d'entablement, actuellement à Saillans dans le mur de la place du Prieuré, à la mairie et chez M. Roche dans la grand-rue, proviennent de $\mathrm{l}^{34}$. Ces fragments présentent des modillons décorés qui soutiennent le bas d'une corniche en doucine; dans les intervalles entre les modillons on remarque, outre une rangée d'oves, des fleurons a quatre ou six pétales découpés et des motifs en croix qui ressemblent à deux boucliers allongés l'un sur l'autre (fig. 4); il subsiste

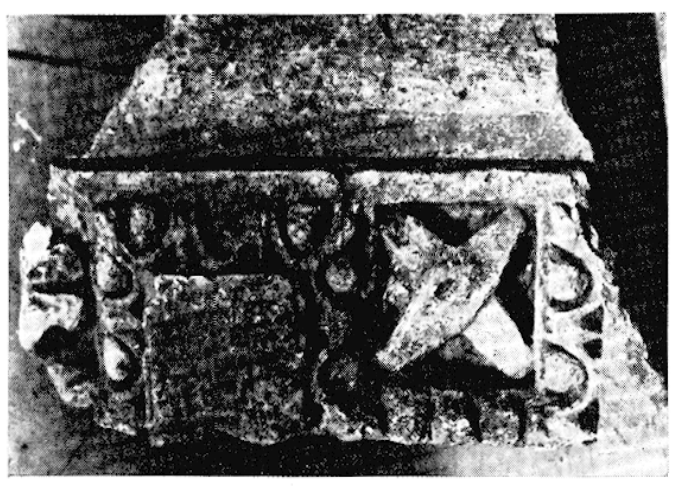

4 Fragment d'entablement déposé à la mairie de Saillans. Cliché A. Häusli.

également un vestige de frise à rinceaux. L'ensemble paraît médiocre; la pierre est un grès calcaire tendre de couleur blanche.

Lors du sondage qui a ramené au jour la seconde partie de l'inscription, on a observé

31 Maurice Pixhard, Topographie du vieux Saillans, dans Bull. Soc. arch. Drôme, LXXVII, 1968, p. 202-203 et 218.

32 Nicolas Choru: 1661, éd. Valence 1878 , p. 130.

33 Jean-Denis Lovg, Recherches sur les antiquilés romaines du pays des Vocontiens, Mémoires présentés à l'Académie des Inscriplions, II, 1849, p. 367.

34 André Mallher, IIistoire de Saillans, Paris 1893 , p. 89, n. 1 que celle-ci élait couchée horizontalement au-dessus d'un massif de maçonnerie épais d'au moins $0,75 \mathrm{~m}$, maçonnerie faite de pierres non taillées, anarchiquement noyées dans du mortier. La surface supérieure en était à cel endroit netlement bombée, formant comme une sorte de petit dôme, et utilisait de nombreux fragments de celle pierre gréseuse dans laquelle on a taillé l'entablement (fig. 5).

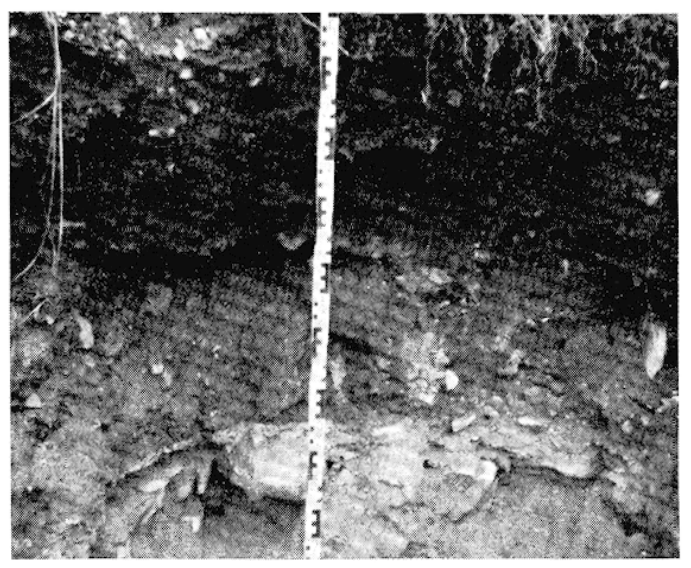

j) Maconneric à surface bombée vue à la Maladreric Ic 3 septembre 1982.

Enlin, en février 1965, on avait repéré à quelques mètres plus au nord, sous le tertre aujourd'hui en partic arasé, un mur est-ouest long de $3,30 \mathrm{~m}$, haut de $1,05 \mathrm{~m}$ et épais de $0,76 \mathrm{~m}$, construit en assises de moellons grossiers.

Indépendamment de ces vestiges, le site présente encore un aulre intérêt : il correspond à la mutatio Darentiaca que signale le Pèlerinage de Bordeaux à Jérusalem, de 333, à sept milles à l'est de la mansio Augusta (Aouste) et à seize milles à l'ouest de la civilas Dea Vocontiorum (Die). En effet, par la route actuelle, directe, il y a $12 \mathrm{~km}$ entre Aouste et le bourg. de Saillans, c'est-à-dire $1650 \mathrm{~m}$ de trop, soit plus d'un mille, par rapport aux sept milles indiqués $(10353 \mathrm{~m})$; on supprime la difficulté en plaçant la mulatio Darentiaca à la Maladrerie même, à $1 \mathrm{~km}$ en direction d'Aouste. En revanche, de ce point a Die on compte $24 \mathrm{~km}$ par le tracé ancien de la roule, soit exactement les 16 milles requis, $23664 \mathrm{~m}$. C'est sans doute de là que proviennent les deux bornes du seizième mille signalées plus haut. 


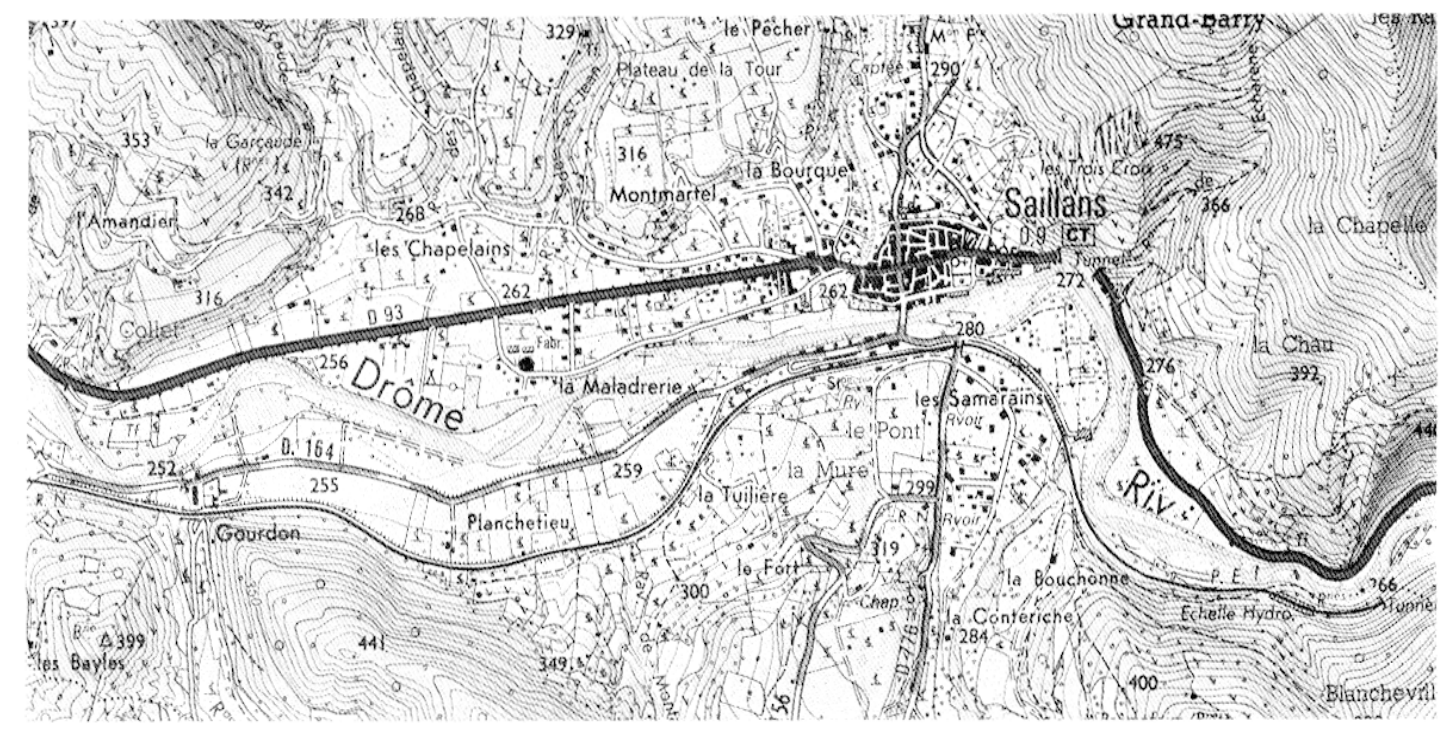

6 Site de la Maladrerie, au centre de la carte. líchelle : 1/25.000.

A la Maladrerie, la voie de Valence à Gap franchissait le ruisseau Saint-.Jean, à vrai dire peu important (fig. 6) ; Maurice Peyrard pense qu'il y avait là également un gué sur la Irôme et un carrefour. Milliaire, gué, carrefour, monument ou tombeau, on retrouve ici un groupement bien caractéristique le long des voies romaines ${ }^{35}$.

Le site correspondait à un domaine en acum, Darentiacum, toponyme connu par le seul Pèlerinage et tiré de l'anthroponyme gaulois Darentius ${ }^{36}$. On peut se demander si c'était là, plus de trois siècles auparavant, la propriété de A. Pompeius Fronto.

Les rapports entre l'inscription, le massif de maçonnerie et l'entablement paraissent bien réels, quoique difficiles à saisir. Les plaques de marbre ont été trouvées près de la maçonnerie ; celle-ci renferme des pierres de même nature que les entablements (on a d'ailleurs récolté dans le sondage un fragment de doucine travaillé dans le même matériau);

35 Sur l'emplacement de Iarentiaca, cf. M. P'ExRARD, op. cit., p. 202-204; IIenri D):SAYk, Apercus sur la campayne disise à l'époque romaine, s!̣e (iongres des socieles savantes, lyon 1964, archeologie, p. 177.

36 A. Dalizar et Ch. Hostasis, Hiclionnaire elymologique des noms de lieux en France, Paris (1963), s.v. Drancy, p. 254. et la sculpture de la mairie présente, a la base du larmier, un tracé incurvé de quelques millimètres. Dans ces conditions, on peut supposer qu'il y avait ici un mausolée circulaire (celui du maître de la villa voisine), couronné par un entablement, du mur duquel seraient tombées les plaques de l'inscription ; la maçonnerie bombée représenterait le socle d'un tertre funéraire, à moins qu'il ne s'agisse tout simplement d'un entassement de matériaux pour four à chaux !

$$
\text { ** }
$$

On voit l'intérêt de l'inscription de Saillans pour la paléographie, l'onomastique, la philologie, l'histoire du site, celle des relations commerciales avec l'Italie et de la romanisation des Voconces septentrionaux vers l'ère chrétienne. Ce texte confirme l'importance de l'aristocratie terrienne au début de l'Empire : plus peut-être qu'à Valence, colonie ancienne ${ }^{37}$, les riches Voconces vivent sur leurs terres ${ }^{38}$.

\section{IIenri DESAYE}

37 André Braxi, folmia lalenlia, Paris 1982, p. 177.

38 Sur les villas de la vallé de la I)rome, ef. H. DESAYE, op. cil., p. 180 el 183. 